\title{
CD Home Nanogrid: Avoiding Standby Consumption and Diminishing Ripple Amplitudes
}

\author{
Miguel Cordova-Fajardo, E Tututi
}

\begin{abstract}
The nanogrids are the basic unit to develop more complex and stronger distributed systems. DC nanogrids allow a better performance and higher efficiency than the AC nanogrids. The lack of DC home appliances in the local market has resulted in a slow development of DC Home Nanogrids (DCHN). The Compact Fluorescent Lamp (CFL) and LED are used in power electronics as high efficiency and low cost lightning components. However, these devices represent a source of emission of harmonics in Alternating Current (AC) and Direct Current (DC) systems, due to their nonlinear behavior. In this paper a mathematical model for the nonlinear loads and experimental results of the voltage and current ripple in a DCHN are presented. The CFL and LED lamps are used as our nonlinear loads for test the model. The model explains well the experimental results of how the ripple amplitude is reduced. We also present the effect of decreasing the ripple amplitude when it is incorporated an induction stove in standby mode to the DCHN, which also is explained by the model.
\end{abstract}

Index Terms-DC nanogrid, appliance, ripple, standby consumption.

\section{INTRODUCTION}

$\mathbf{T}$ HE nanogrids are the basic elements of larger systems such as micro grids or grids, which result very important to achieve real electric distributed systems. In fact, the relevance of a home nanogrid stems from its capacity to generate electric energy and the possible incorporation of Renewable Energy Sources (RES) on it. The addition of energy storage devices in a home nanogrid is crucial to reduce the dependence of energy of the grid, this storage devices could be also of great importance in rural areas [1], [2]. The advantages that DCHN's present are, for example, the efficiency, the performance and their simply control, among others, giving the end user the possibility of participate in the energetic market. The grow in the use of DCHN has been slow due to the lack of DC home appliances in the local market and the dominance of $\mathrm{AC}$ home appliances, even in the case when they can operate in DC [2]. The common use of appliances in the DCHN is to prepare and cooking food as well as lightning and refrigeration or communication, among others. The energy efficiency standards have been established to regulate the energy consumption and efficiency of the home appliances, such as Energy Star [3]. The incorporation of high efficiency appliances to the nanogrid, is related with noise emission and pollution into the grid emitted by the switching

Miguel Cordova-Fajardo is with Instituto Tecnológico de Lázaro Cárdenas, Lázaro Cárdenas, Michoacán, México. (e-mail: mangelcordova@1cardenas.tecnm.mx ).

E. S. Tututi is with Universidad Michoacana de San Nicolás de Hidalgo, Morelia, Michoacán, Mexico. (e mail: tututi@umich.mx ) power supplies used in the appliances [4]. The CFL's and Led lamps, and other home appliances are associated with the voltage and current distortion in AC systems [5], [6]. Most of studies in the literature are focused in the analysis and modeling the behavior of switching power supplies [7], [8], since substituting the circuit of a switching power supply (SPS) for an equivalent circuit, it results a nontrivial process. However, in the case of the DCHN, low attention has been paid in the behavior of appliances and their modeling. As a part of the analysis of a DCHN, we are interesting in the behavior of voltage and current in the system instead of the components and the effects of the SPS.

In this paper a nonlinear mathematical model for the loads in a DCHN is presented. The propose of the model is to describe the behavior of AC home appliances in the DCHN. It is important to mention that many appliances operate with an internal DC voltage. For our analysis, we resort to [9], where were analyzed the effects of CFL and LED lamps in a DC nanogrid. The voltage used in the DCHN was of $120 \mathrm{VDC}$, with variations from 100 to 140 VDC. The energy generation sector was conformed by 5 photovoltaic panels (PV), and an energy storage sector containing 10 batteries. Finally, in the load sector were incorporated appliances and a DC bus [10].

Several studies have been carried out of how to diminish the distortion waveform also called harmonics, due to the nonlinear loads. The analogous on DC systems are usually observed as voltage and current ripples. The solutions presented in AC systems consist in to designed and use an Electromagnetic Interference Filter (EMI) [11]-[13]. These filters can produce energy consumption in the state denominated as standby mode. This mode is presented when the home appliance in question spends energy without making any real work.

An outline of this paper is as follows. In Sect. II we present the mathematical model to the non linear load. In Sect. III the results of the experimental measurements are presented. In Sect. IV we discus our results and compare them with the numerical simulations. Finally, in Sect.V we give our conclusions.

\section{MAThematicAl MODEL}

In this section we present a general model for nonlinear loads in a DCHN, where the internal components are in principle unknown. However, their behavior can be described by using continuous periodical functions, which make possible reproduce the experimental data. When we design a DCHN it is important to know the features of each component in different sectors of the system. For the energy storage sector, 


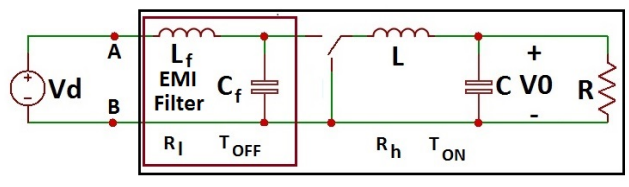

Fig. 1. Buck converter.

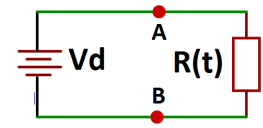

Fig. 2. Nonlinear load incorporated into DCHN.

the batteries are modeled by the function $V_{B a t}(t)=V_{d}$ where $V_{d}$ is a constant.

For the loads sector, it is common to consider linear loads expressed by $R(t)=R$, being $R$ a constant. However, high efficiency home appliances that are used in the DCNH employ SPS's producing low energy consumption, but the appliances result to be non linear loads. Thus, the loads can not be represented simply by a constant function $R$. Instead, a more elaborated function is necessary in order to model each appliance in the DCHN. One way of face this issue is to look for a model as simple as possible which gives a mathematical description of nonlinear loads in an equivalent simple circuit.

In order to obtain stability on microgrids, one looks for control of voltage and current as simplest as possible in each DCHN [14], [15], However, due to the nonlinear behavior of the loads incorporated into the DCHN (DC systems) makes difficult the analysis of the electric parameters in the grid. There are several models in the literature that tackle this issue [16]-[18]. For instance, the behavior of the AC voltage supplies, can be model by $V(t)=V_{m} \sin (\omega t)$ as a main feature of the system, where $V_{m}$ is the amplitude of the voltage and $\omega$ is the fundamental frequency in the system. In some cases the parameters mentioned for this model are enough to determine experimental measurements, for example the impedance $Z$. On the other hand, a SPS presents a periodic behavior in which a time $T_{O N}$ corresponds to the maximal energy consumption while the time $T_{O F F}$ occurs when the energy consumption is minimal, being $T=T_{O N}+T_{O F F}$ the period of the system. Thus, the switching frequency is simple $\omega_{s}=2 \pi / T$. Let us perform an analysis of the nonlinear loads viewing them as black boxes. For this, we have considered a buck converter circuit shown in Fig 1 . At the time $T_{O F F}$, the active elements in the buck converter are the corresponding to the EMI filter components. The next state corresponds to $T_{O N}$ and so on. In this case the transistor feds the $R C L$ circuit, which keeps a constant voltage $V_{0}$ at the resistance $R$. The EMI filter is always connected to the $A$ and $B$ terminals.

In order to model a nonlinear load, we propose the function $R(t)$ as a mathematical model which replaces the nonlinear load at the $A$ and $B$ terminals, resulting the simple configuration shown in the Fig. 2. We have tested different functions to model the loads finding that the periodic functions adjust

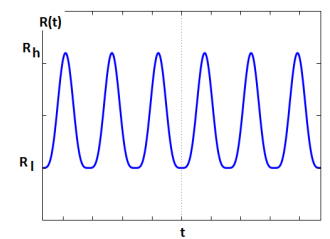

Fig. 3. Nonlinear load R(t).

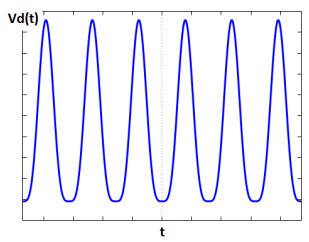

Fig. 4. Voltage ripple model.

well to the experimental results. An economical proposal to the function $R(t)$ is:

$$
R(t)=R_{h} \sin ^{n}\left(2 \omega_{s} t\right)+R_{l},
$$

which describes a similar behavior of the SPS. Respect to Fig. 1, it is possible to establish a relation between $R_{l}$ and the EMI filter, while $R_{h}$ is related with both the EMI filter and the $R C L$ circuit. The sinus function gives the periodic transitions between $R_{l}$ and $R_{h}$ values of the load. Notice that the cosine function can also be used, however we prefer the sine function because it gives the proper initial conditions. In order to establish the model, let us observe that as $n$ becomes large enough, $T_{O F F}$ increases while $T_{O N}$ decreases, which will be used to choose the $n$ value.

The batteries set has been represented with the supply $V_{d}(t)$ that feeds DC voltage to the DCHN. Thus, the instantaneous power is given by:

$$
P=V_{d}(t) I_{B a t}(t),
$$

where $P$ stands for a predefined constant. The battery current $I_{\text {Bat }}(t)$ in Fig. 2 can be calculated from the Ohm law:

$$
I_{\text {Bat }}(t)=\frac{V_{d}(t)}{R(t)},
$$

from which we obtain the voltage $V_{d}(t)$, given by:

$$
V_{d}(t)=\sqrt{P R(t)},
$$

which determines the voltage in the DCHN. In order to consider the switching time such that $T_{O F F}$ is slightly bigger than $T_{O N}$ and a load described through an even function, we take simple $n=4$ in Eq. 1, resulting

$$
R(t)=R_{h} \sin ^{4}\left(2 \omega_{s} t\right)+R_{l} .
$$

The load $R(t)$ generates a ripple in the voltage and current for which their functions is shown in Fig. 4. Notice that the voltage waveform presents a periodical behavior, and its frequencies is the same as the corresponding nonlinear load $R(t)$. 


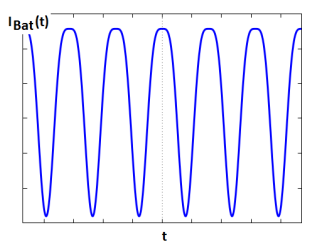

Fig. 5. Current ripple model.

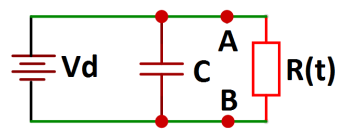

Fig. 6. Capacitor incorporated in DCHN.

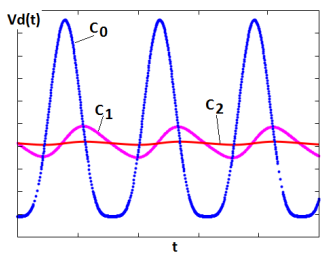

Fig. 7. Voltage ripple in DCHN. The load is represented by means of a sinusoidal even function.

With the propose of reducing large amplitudes in the current and voltage ripple, it is incorporated a filter capacitor $C$ into the schematic circuit shown in Fig. 6. In this case, by applying Kirchhoff laws, the circuit is described by the equations:

$$
\begin{gathered}
V_{d}(t)=V_{C}(t)=V_{R}(t), \\
I_{\text {Bat }}(t)=I_{C}(t)+I_{R}(t) .
\end{gathered}
$$

Now, the battery current $I_{B a t}(t)$ is:

$$
\begin{aligned}
I_{B a t}(t) & =C \frac{d V_{C}(t)}{d t}+\frac{V_{R}(t)}{R(t)}, \\
& =C \frac{d V_{d}(t)}{d t}+\frac{V_{d}(t)}{R(t)} .
\end{aligned}
$$

By using Eqs. (2) and (8), we obtain the derivative of the voltage in the battery

$$
C \frac{d V_{d}(t)}{d t}=\frac{P}{V_{d}(t)}-\frac{V_{d}(t)}{R(t)} .
$$

Notice that, when $C=0$, Eq. (9) is reduced to the Eq. (4). Applying the Runge-Kutta of order fourth to solve numerically Eq. (9), we obtain the result for the voltage, which is illustrated in Fig. 7. The cases $C_{2}>C_{1}>C_{0}=0$ have been considered, with $C_{2}=10 C_{1}$. It can observed that the ripple amplitude diminishes as the capacitance $C$ grows, which is corroborated with the experimental results, as it will be shown below.

Let us now discuss the model for $R(t)$ by using a sinusoidal odd function:

$$
R(t)=R_{h} \sin ^{13}\left(2 \omega_{s} t\right)+R_{l}
$$

whose behavior is displayed in Fig. 8. In a similar way, we can

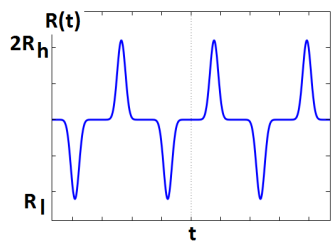

Fig. 8. The load is represented by means of a sinusoidal odd function.

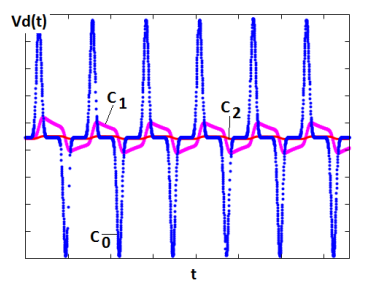

Fig. 9. Voltage ripple, odd function.

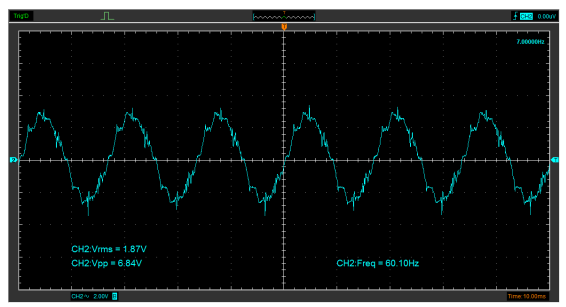

Fig. 10. The standby consumption of the induction stove at AC is $1.87 \mathrm{~A}$.

carry out an analysis for this load. In fact, from Eq. (9) we can obtain the numerical solution. Once again, we get a voltage ripple as it is presented in Fig. 9, also for three different cases: $C_{2}>C_{1}>C_{0}=0$. We observe again the fact that the ripple amplitude diminishes as the capacitor value becomes larger. In next section it will be presented experimental results for the voltage and current ripple when two kinds of lighting devices (CFL and LED Lamps) are included in the DCHN. The standby consumption of the induction stove in an AC system will be presented as well.

\section{EXPERIMENTAL STUDY}

The possibility of incorporating AC lighting devices into a DCHN has been previously studied [9]. These devices are related with emission of harmonics in AC systems [19]. In this section, we present experimental results of voltage and current ripples, as well as the standby consumption in a DCHN due to lighting devices and an induction stove, respectively.

The induction stove consumes relatively great quantity of energy in standby mode when it is connected to the voltage (in our case, $127 \mathrm{VAC}$ ). In this mode, the stove presents energy losses that have been measured through the current which, in this case, gives a value of $1.87 \mathrm{~A}$. This consumption is due to presence of a filter capacitor inside the appliance whose value is $40 \mu \mathrm{f}$. The waveform of the current is shown in Fig. 10.

Any induction stove can be incorporated directly into a DCHN without modification. In fact, the internal voltage needed to operate is $\mathrm{DC}$; when it is fed by $\mathrm{AC}$ voltage, 


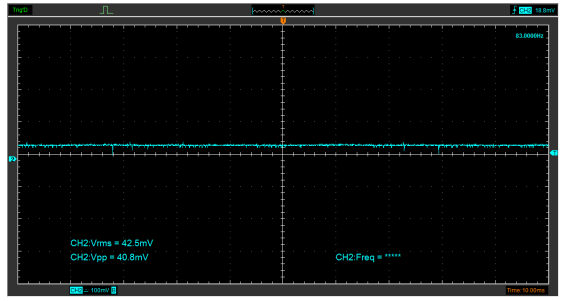

Fig. 11. The standby consumption of the induction stove in DCHN.

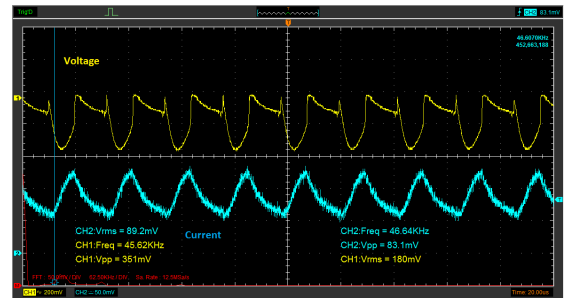

Fig. 12. CFL voltage and current ripple in the DCHN.

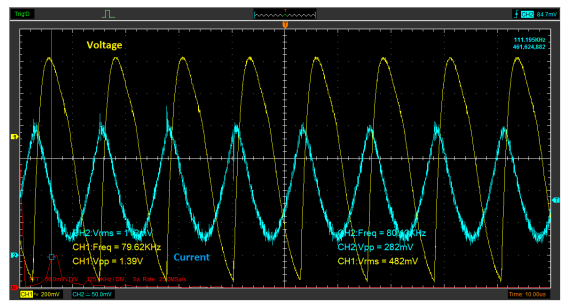

Fig. 13. LED voltage and current ripple in the DCHN.

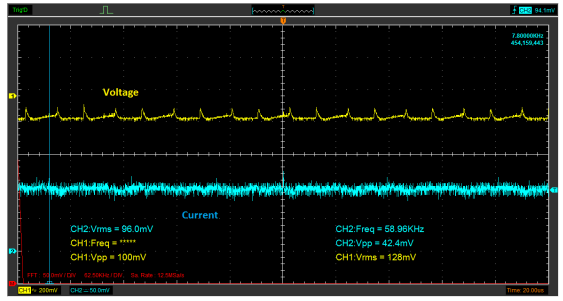

Fig. 14. Attenuated CFL voltage and current ripple.

this is rectified by switching supplies. On the other hand, the standby consumption of the induction stove when operates in the DCHN is almost null. Its standby current is shown in Fig. 11.

The voltage and current ripple when the CFL and LED are operating in the DCHN are presented in Figs. 12 and 13, respectively. The CFL voltage ripple is $351 \mathrm{mV}$ Pk-Pk, while the current ripple is $83.1 \mathrm{~mA} \mathrm{Pk-Pk}$. The LED voltage ripple is $1.39 \mathrm{~V} \mathrm{Pk-Pk}$, and its current ripple is $282 \mathrm{~mA} \mathrm{Pk}-$ $\mathrm{Pk}$. The simultaneous use of several home appliances in the electric system is frequent. For this case, the Figs. 14 and 15 present the experimental graphs corresponding to the voltage and current ripple of the DCHN due to CFL and LED lamps, respectively. The data were taken when the induction stove was in standby mode while the lighting device was working. Notice that the voltage and current ripple become smaller due the effect of the filter capacitor in the induction stove.

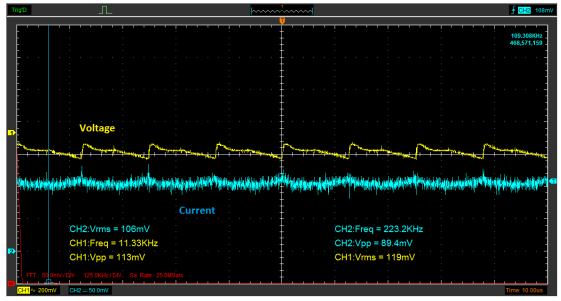

Fig. 15. Attenuated voltage and current ripple in the LED.

\section{ANALYSIS}

Let us compare the results of the proposed models for the load $R(t)$ with the experimental results. The behavior of the voltage of the LED from experimental data, shown in Fig. 13, is quite similar to the obtained by using the model, presented in Fig. 4. In this case, the load model is represented in terms of an even function. Whereas, the CFL voltage ripple shown in Fig. 12 has a similar shape for the case of the $C_{1}$ presented in Fig. 9. For this instance, we used an odd sinusoidal function model for the load $R(t)$. We should remark that, in last case, the waveform obtained by taking $C_{0}=0$ in our model, is not present in the experimental result, due to existence of a filter capacitor in the CFL. The solution of Eq. (9) shows a diminishing in the voltage ripple when the capacity $C$ is increased. The simultaneous operation of the induction stove and one lamp has been also evaluated, given an extra reducing in the ripple amplitude. The standby consumption of the induction stove working in $\mathrm{AC}$ system was of $1.8 \mathrm{~A}$, while at DCHN was of $42.5 \mathrm{~mA}$.

The lamps devices are sources of harmonics, and their effects result in the voltage and current ripples of the DCHN. In each lamp, we observe a periodical function in voltage and current waveform. From Fig. 12, we can identify a range of frequencies from 45.62 to $46.64 \mathrm{KHz}$ in the CFL voltage and current ripples. For the LED lamp, we obtained a range of frequencies from 79.62 to $80.40 \mathrm{KHz}$ (see Fig. 13). When the induction stove is connected and remains in standby mode, we observe that the behavior of the ripple in the DCHN changes due to the presence of the CFL or the LED lamp. The CFL voltage ripple has been reduced to $100 \mathrm{mV}$ Pk-Pk where the diminishing is of $71.50 \%$ with respect to the initial configuration (when the stove is not connected). The CFL current ripple is reduced to $42.4 \mathrm{~mA} \mathrm{Pk-Pk}$, which represents a diminishing of the $48.97 \%$ with respect to the initial value. The highest diminishing occurs in the LED voltage ripple being of $91.87 \%$; their final voltage is of $113 \mathrm{mV}$ Pk-Pk, and current ripple is reduced to $89.4 \mathrm{~mA}$ Pk-Pk.

\section{CONCLUSIONS}

The electronic devices incorporate filter capacitors into their components which diminish electromagnetic and noise interference in AC systems. Although the fundamental frequency of the DCHN is zero, we have observed ripples with high frequencies that are emitted from the lighting devices into the DCHN. These high frequencies correspond to typical switching frequencies of the SPS. The effects of ripples on different sectors of the DCHN are yet unknown. However, the 
diminishing of ripple amplitudes can be controlled. In fact, the main reason of this reducing is due to the effect of a filter capacitor $C$, as it is corroborated with our mathematical model and the experimental results. The capacitor can contribute to a better stability of the DCHN and can also be an energy storage element. On the other side, the standby consumption of the induction stove in DCHN is practically reduced to zero. The nonlinear load models are proposed in base to experimental behavior of the DCHN. However, the model is made up in terms of an equivalent circuit for a real nonlinear load, where the parameters $R_{h}$ and $R_{l}$ must be adjusted by the features of the DCHN according to SPS used. Through the model we have obtained a qualitative description of the voltage waveform corresponding to two different loads at the stationary state. Finally, the incorporation of RES into the electric grid has allowed the growth of distributed electric systems, let us stress that the energy storage devices will allow a rapid growth in the use of the DCHN's and reduce the dependence of fossil fuels. This makes necessary the establishment of general standards for the DCHN, and the design of improved circuits to enlarge the life cycle in each sector of the DCHN, as well as general parameters for the DC home appliances.

\section{ACKNOWLEDGMENT}

The authors thanks to CONACYT, CIC-UMSNH and Tecnológico Nacional de México for financial support.

\section{REFERENCES}

[1] N. Munzke, B. Schwarz, F. Büchle, and M. Hiller, "Evaluation of the efficiency and resulting electrical and economic losses of photovoltaic home storage systems," Journal of Energy Storage, vol. 33, p. 101724.

[2] D. Villanueva, M. Cordeiro, A. Feijóo, E. Míguez, and A. Fernández, "Effects of adding batteries in household installations: Savings, efficiency and emissions," Applied Sciences, vol. 10, no. 17, p. 5891, 2020.

[3] A. M. Ohler, D. G. Loomis, and K. Ilves, "A study of electricity savings from energy star appliances using household survey data," Energy Policy, vol. 144, p. 111607, 2020.

[4] J. A. Suarez, G. di Mauro, D. Anaut, and C. Aguero, "Analysis of the harmonic distortion and the effects of attenuation and diversity in residential areas," IEEE Latin America Transactions, vol. 3, pp. 53-59, Dec 2005

[5] N. Phannil, C. Jettanasen, and A. Ngaopitakkul, "Harmonics and reduction of energy consumption in lighting systems by using led lamps," Energies, vol. 11, no. 11, p. 3169, 2018.

[6] G. Georgescu and B. Neagu, "The pollution with harmonics in public electric energy repartition and distribution system," in Proc. of ISEEEC, pp. 251-256, 2009.

[7] A. B. Nassif, J. Yong, W. Xu, and C. Chung, "Indices for comparative assessment of the harmonic effect of different home appliances," International Transactions on Electrical Energy Systems, vol. 23, no. 5, pp. 638-654, 2013.

[8] V. George, A. Bagaria, P. Singh, S. R. Pampattiwar, and S. Periwal, "Comparison of cfl and led lamp-harmonic disturbances, economics (cost and power quality) and maximum possible loading in a power system," in 2011 International Conference \& Utility Exhibition on Power and Energy Systems: Issues and Prospects for Asia (ICUE), pp. 1-5, IEEE, 2011.

[9] M. A. Cordova-Fajardo and E. S. Tututi, "How the fluorescent and led lamps affect the dc home nanogrids," in 2020 IEEE Conference on Technologies for Sustainability (SusTech), pp. 1-5, 2020.

[10] M. A. Cordova-Fajardo and E. S. Tututi, "Incorporating home appliances into a DC home nanogrid.," Journal of Physics: Conference Series, vol. 1221, p. 012048, jun 2019.

[11] P. Fang, B. White, C. Fiorentino, and Y.-F. Liu, "Zero ripple single stage ac-dc led driver with unity power factor," in 2013 IEEE Energy Conversion Congress and Exposition, pp. 3452-3458, IEEE, 2013.
[12] J. Liao, N. Zhou, and Q. Wang, "Design of low-ripple and fast-response dc filters in dc distribution networks," Energies, vol. 11, no. 11, p. 3128 , 2018.

[13] Ó. Lucía, I. Cvetkovic, H. Sarnago, D. Boroyevich, P. Mattavelli, and F. C. Lee, "Design of home appliances for a dc-based nanogrid system: An induction range study case," IEEE Journal of Emerging and Selected Topics in Power Electronics, vol. 1, no. 4, pp. 315-326, 2013.

[14] M. Nasir, Z. Jin, H. A. Khan, N. A. Zaffar, J. C. Vasquez, and J. M. Guerrero, "A decentralized control architecture applied to dc nanogrid clusters for rural electrification in developing regions," IEEE Transactions on Power Electronics, vol. 34, no. 2, pp. 1773-1785, 2019.

[15] M. Mosayebi, S. M. Sadeghzadeh, J. M. Guerrero, and M. H. Khooban, "Stabilization of dc nanogrids based on non-integer general type-ii fuzzy system," IEEE Transactions on Circuits and Systems II: Express Briefs, vol. 67, no. 12, pp. 3108-3112, 2020.

[16] A. Francés, R. Asensi, O. García, R. Prieto, and J. Uceda, "A blackbox modeling approach for dc nanogrids," in 2016 IEEE Applied Power Electronics Conference and Exposition (APEC), pp. 1624-1631, IEEE, 2016.

[17] G. Arunkumar, D. Elangovan, P. Sanjeevikumar, J. B. H. Nielsen, Z. Leonowicz, and P. K. Joseph, "Dc grid for domestic electrification," Energies, vol. 12, no. 11, p. 2157, 2019.

[18] V. Chekh, D. Lyapunov, and A. Pravikova, "Mathematical model of power supply system for remotely operated underwater vehicle with dc power transmission line and load voltage feedback," in IOP Conference Series: Materials Science and Engineering, vol. 1019, p. 012028, IOP Publishing, 2021.

[19] C. Jiang, D. Salles, W. Xu, and W. Freitas, "Assessing the collective harmonic impact of modern residential loads part ii: Applications," IEEE Transactions on Power Delivery, vol. 27, no. 4, pp. 1947-1955, 2012. 